\title{
A Selective Chronology of Historical and Literary Events
}

\begin{tabular}{|c|c|c|c|}
\hline Year & Regions & Historical Event & Literary Event \\
\hline 756 & Spain & $\begin{array}{l}\text { Abd ar-Rahman's emirate } \\
\text { in Cordova }\end{array}$ & \\
\hline 793 & England & $\begin{array}{l}\text { Vikings plunder Lindisfarne } \\
\text { convent }\end{array}$ & \\
\hline 874 & Iceland/Faeroe Islands & $\begin{array}{l}\text { Norwegians settle in Iceland } \\
\text { and Faeroe Islands }\end{array}$ & \\
\hline 875 & $\begin{array}{l}\text { Greenland/North } \\
\text { Atlantic }\end{array}$ & Gunnbjørn in Greenland & \\
\hline 878 & England & Danelaw in England & \\
\hline $912-61$ & Spain & Caliphate in Cordova & \\
\hline 1013 & England & $\begin{array}{l}\text { Swein Forkbeard conquers } \\
\text { England (ruled by Danes } \\
\text { until 1042) }\end{array}$ & \\
\hline 1066 & England & $\begin{array}{l}\text { William the Conqueror } \\
\text { beats Harald Godvinson } \\
\text { at Hastings }\end{array}$ & \\
\hline 1071 & Italy & Norse conquer southern Italy & \\
\hline c. $1170-1400$ & Iceland/North Atlantic & Icelandic and Norse sagas & \\
\hline $1271-95$ & Asia & $\begin{array}{l}\text { Marco Polo's journeys } \\
\text { to China }\end{array}$ & \\
\hline 1299 & Middle East & $\begin{array}{l}\text { Osman Empire founded by } \\
\text { Osman I }\end{array}$ & \\
\hline c. 1300 & Middle East & Osman Empire in Asia Minor & \\
\hline 1345 & Middle East/Europe & $\begin{array}{l}\text { Ottoman Turks make their first } \\
\text { crossing into Europe }\end{array}$ & \\
\hline $1380-1814$ & North Europe & Denmark and Norway united & \\
\hline 1415 & Portugal/Africa & $\begin{array}{l}\text { Portuguese capture Ceuta } \\
\text { (North Africa) }\end{array}$ & \\
\hline 1427 & Atlantic & $\begin{array}{l}\text { Discovery of Azores by } \\
\text { Portuguese }\end{array}$ & \\
\hline 1441 & Portugal/Africa & Start of slave trade & \\
\hline 1445 & Portugal/Africa & $\begin{array}{l}\text { Portuguese reach Cape } \\
\text { Verde }\end{array}$ & \\
\hline 1453 & Middle East & $\begin{array}{l}\text { Constantinople conquered by } \\
\text { Ottoman Turks }\end{array}$ & \\
\hline 1471 & Portugal/Africa & Portuguese on Gold Coast & \\
\hline 1473 & North Atlantic & $\begin{array}{l}\text { Kristian I organises } \\
\text { expeditions to Greenland and } \\
\text { Labrador }\end{array}$ & \\
\hline 1482 & Portugal/Africa & First voyage of Diogo Cão & \\
\hline
\end{tabular}




\begin{tabular}{|c|c|c|c|}
\hline Year & Regions & Historical Event & Literary Event \\
\hline 1487 & Portugal/Africa & $\begin{array}{l}\text { Bartolomeu Dias rounds Cape } \\
\text { of Good Hope }\end{array}$ & \\
\hline 1492 & Europe & $\begin{array}{l}\text { Spanish conquest of Granada } \\
\text { Expulsion of Jews from Spain }\end{array}$ & \\
\hline 1492 & The Americas & Columbus reaches 'New World' & \\
\hline 1493 & Caribbean & Settlement of Hispaniola & \\
\hline 1494 & South America & $\begin{array}{l}\text { Portugal and Castile partition } \\
\text { exploration and exploitation } \\
\text { of the world (East and West } \\
\text { of c. } 50 \text { degrees meridian resp.) }\end{array}$ & \\
\hline $1497-98$ & Asia & $\begin{array}{l}\text { Vasco da Gama makes first } \\
\text { Portuguese voyage to and from } \\
\text { India }\end{array}$ & \\
\hline 1499 & South America & $\begin{array}{l}\text { Italian navigator Amerigo } \\
\text { Vespucci sights coast of South } \\
\text { America }\end{array}$ & \\
\hline 1500 & South America & $\begin{array}{l}\text { Portuguese navigator Pedro } \\
\text { Álvares Cabral accidentally } \\
\text { reaches and then seizes Brazil }\end{array}$ & \\
\hline 1503 & Caribbean & First sugar mill in Hispaniola & \\
\hline 1505 & Asia & Portuguese in Ceylon & \\
\hline 1510 & India & $\begin{array}{l}\text { Afonso de Albuquerque } \\
\text { conquers city of Goa }\end{array}$ & \\
\hline $1510-50$ & The Americas & $\begin{array}{l}\text { Spanish ship large quantities } \\
\text { of gold from New World to } \\
\text { Spain }\end{array}$ & \\
\hline 1511 & The Americas & $\begin{array}{l}\text { First Spanish settlement on } \\
\text { American mainland }\end{array}$ & \\
\hline 1511 & Asia & Portuguese in Java and Malacca & \\
\hline 1512 & The Americas & $\begin{array}{l}\text { Arrival of first bishop from } \\
\text { Europe in the Americas }\end{array}$ & \\
\hline 1513 & The Americas & $\begin{array}{l}\text { Spanish conquistador Vasco } \\
\text { Núñes de Balboa discovers } \\
\text { Pacific Ocean }\end{array}$ & \\
\hline 1514 & South America & $\begin{array}{l}\text { Spanish missionary Bartolomé } \\
\text { de Las Casas begins work in } \\
\text { support of better treatment of } \\
\text { indigenous peoples of the } \\
\text { Americas }\end{array}$ & \\
\hline 1516 & South America & $\begin{array}{l}\text { Portuguese begin sugar } \\
\text { cultivation in Brazil }\end{array}$ & \\
\hline 1516 & South America & & $\begin{array}{l}\text { Garcia de Resende, } \\
\text { Cancioneiro Geral }\end{array}$ \\
\hline $1519-21$ & Central America & $\begin{array}{l}\text { Cortés and his Spaniards destroy } \\
\text { Aztec empire and begin building } \\
\text { Mexico City }\end{array}$ & \\
\hline 1521 & Asia & $\begin{array}{l}\text { Portuguese Ferdinand Magellan } \\
\text { explores islands now known as }\end{array}$ & \\
\hline
\end{tabular}




\begin{tabular}{|c|c|c|c|}
\hline Year & Regions & Historical Event & Literary Event \\
\hline & & $\begin{array}{l}\text { Philippines, approaching them } \\
\text { from Pacific Ocean }\end{array}$ & \\
\hline 1524 & North America & $\begin{array}{l}\text { Giovanni da Verrazano lands } \\
\text { in Carolinas and explores } \\
\text { northwards }\end{array}$ & \\
\hline 1526 & Africa & $\begin{array}{l}\text { Congolese king Mbemba } \\
\text { Nzinga protests to Portugal's } \\
\text { Joao III that Portuguese } \\
\text { merchants are selling his people } \\
\text { as slaves to Brazilian sugar } \\
\text { planters }\end{array}$ & \\
\hline 1529 & Europe & $\begin{array}{l}\text { Siege of Vienna } \\
\text { Ottoman expansion in } \\
\text { Central Europe stopped }\end{array}$ & \\
\hline $1532-6$ & South America & Pizarro conquers Incas & \\
\hline $1530 s$ & South America & $\begin{array}{l}\text { Spanish conquest of } \\
\text { New Granada (Colombia) }\end{array}$ & \\
\hline 1532 & & & $\begin{array}{l}\text { Francisco de Vitoria, } \\
\text { Doctrina sobre los } \\
\text { indios }\end{array}$ \\
\hline 1534 & North America & $\begin{array}{l}\text { Jacques Cartier undertakes first } \\
\text { voyage to New France }\end{array}$ & \\
\hline 1536 & South America & $\begin{array}{l}\text { Buenos Aires established by } \\
\text { Spanish }\end{array}$ & \\
\hline 1537 & & Jesuit Order founded & \\
\hline 1541 & South America & Spanish settlement of Chile & \\
\hline 1542 & Far East & $\begin{array}{l}\text { First European contact } \\
\text { (Portuguese) with Japan }\end{array}$ & \\
\hline 1542 & Asia & $\begin{array}{l}\text { Spanish name Philippines } \\
\text { islands and claim them as } \\
\text { colonies }\end{array}$ & \\
\hline 1549 & Asia & Jesuits in Japan & \\
\hline 1552 & Spanish Caribbean & & $\begin{array}{l}\text { Bartolomé de las } \\
\text { Casas, Brevísima } \\
\text { relación de la } \\
\text { destrucción de las } \\
\text { Indias }\end{array}$ \\
\hline 1552 & Portugal & & $\begin{array}{l}\text { Luís de Camões, The } \\
\text { Lusiads }\end{array}$ \\
\hline 1557 & Asia & Portuguese in Macau & \\
\hline $1560 \mathrm{~s}$ & The Americas & $\begin{array}{l}\text { First epidemic of European- } \\
\text { borne diseases in Brazil }\end{array}$ & \\
\hline 1564 & North America & $\begin{array}{l}\text { Spanish missionaries introduce } \\
\text { grapes to California }\end{array}$ & \\
\hline 1565 & North America & $\begin{array}{l}\text { Florida colonised by Pedro } \\
\text { Menendez de Aviles }\end{array}$ & \\
\hline 1565 & Asia & $\begin{array}{l}\text { First Spanish settlement in } \\
\text { Philippines }\end{array}$ & \\
\hline
\end{tabular}




\begin{tabular}{|c|c|c|}
\hline Year & Regions & Historical Event \\
\hline 1570 & Portugal/Africa & $\begin{array}{l}\text { Portuguese establish a colony } \\
\text { in Angola }\end{array}$ \\
\hline 1571 & Europe & $\begin{array}{l}\text { Battle of Lepanto } \\
\text { Holy League fleet defeats } \\
\text { Ottoman fleet }\end{array}$ \\
\hline 1572 & Asia & $\begin{array}{l}\text { Establishment of Manila, as a } \\
\text { port to facilitate shipping of } \\
\text { goods from Asia across Pacific } \\
\text { to Mexico and thence to Spain }\end{array}$ \\
\hline 1573 & & Franciscans arrive in Florida \\
\hline 1574 & Spanish Empire & \\
\hline
\end{tabular}

Battle of Alcácer Quibir Literary Event

Death of Portugal's King

Sebastião

Spanish dominion over

Portuguese territories

c. 1585 Mexico

Diego Muñoz

Camargo, Historia

de Tlaxcala

1588

Europe

1595

Holland/Asia

c. 1598

South America

Defeat of Spanish Armada by

British

Dutch explorer Cornelis de Houtmanpice discovers a new sea route to Indonesia: beginning of Dutch spice trade

Juan Ginés de

Sepúlveda,

Demócrates

Segundo o de las justas causas de la guerra contra los indios

de Tlaxcala

Dioses y hombres de

Huarochirí.

Narración quechua recogida por Francisco de Avila

Holland

North America

Dutch East India Company

founded

Quebec founded by Samuel de Champlain

North America

Dutch East India Company sponsors Henry Hudson's exploration of North America

Tomas Pinpin prints the first book to be published in the Philippines 


\begin{tabular}{|c|c|c|c|}
\hline Year & Regions & Historical Event & Literary Event \\
\hline 1611 & Europe & $\begin{array}{l}\text { Foundation of French East } \\
\text { India Company }\end{array}$ & $\begin{array}{l}\text { Shakespeare's The } \\
\text { Tempest first } \\
\text { performed in London }\end{array}$ \\
\hline 1614 & Japan & $\begin{array}{l}\text { Christian missionaries } \\
\text { expelled from Japan }\end{array}$ & \\
\hline 1614 & Portugal & & $\begin{array}{l}\text { Fernão Mendes } \\
\text { Pinto, The Travels of } \\
\text { Mendes Pinto } \\
\text { (Portuguese) }\end{array}$ \\
\hline 1615 & Asia & $\begin{array}{l}\text { Netherlands seize Moluccas } \\
\text { from Portugal } \\
\text { English defeat a Portuguese } \\
\text { armada off coast of Bombay }\end{array}$ & \\
\hline $1618-48$ & Europe & Thirty Years War & \\
\hline 1619 & North America & $\begin{array}{l}\text { Dutch bring first slaves from } \\
\text { Africa to North America }\end{array}$ & \\
\hline 1620 & Europe/Asia & $\begin{array}{l}\text { Tranquebar in southern India } \\
\text { becomes a Danish colony } \\
\text { (sold to Britain in 1845) }\end{array}$ & \\
\hline 1621 & Holland & $\begin{array}{l}\text { Formation of Dutch West India } \\
\text { Company }\end{array}$ & \\
\hline 1635 & Caribbean & $\begin{array}{l}\text { French claim Martinique and } \\
\text { Guadeloupe }\end{array}$ & \\
\hline $1641-8$ & Dutch Empire & Dutch occupy Luanda & \\
\hline 1652 & Africa & $\begin{array}{l}\text { Dutch establish colony at } \\
\text { Cape of Good Hope }\end{array}$ & \\
\hline 1654 & Central America & Dutch leave Brazil & \\
\hline 1671 & Caribbean & $\begin{array}{l}\text { St Thomas becomes Danish } \\
\text { colony }\end{array}$ & \\
\hline 1673 & Asia & $\begin{array}{l}\text { French trading post established } \\
\text { at Pondicherry }\end{array}$ & \\
\hline 1683 & Europe & $\begin{array}{l}\text { Battle of Vienna } \\
\text { Beginning of decline of } \\
\text { Ottoman Empire }\end{array}$ & \\
\hline 1685 & Caribbean & & $\begin{array}{l}\text { Publication of } \\
\text { Code noir }\end{array}$ \\
\hline 1695 & Caribbean & $\begin{array}{l}\text { Spain cedes Hispaniola to } \\
\text { French }\end{array}$ & \\
\hline $1703-4$ & Asia: Philippines & & $\begin{array}{l}\text { Aquino de Belen, } \\
\text { Pasyon }\end{array}$ \\
\hline 1717 & Atlantic & $\begin{array}{l}\text { English and French slave } \\
\text { trading companies begin slave } \\
\text { trade with Spanish colonies in } \\
\text { Americas }\end{array}$ & \\
\hline 1718 & Caribbean & St John becomes Danish colony & \\
\hline 1721 & Greenland & $\begin{array}{l}\text { Hans Egede arrives in } \\
\text { Greenland to begin } \\
\text { (re)colonisation }\end{array}$ & \\
\hline
\end{tabular}




\begin{tabular}{|c|c|c|c|}
\hline Year & Regions & Historical Event & Literary Event \\
\hline 1733 & Caribbean & $\begin{array}{l}\text { Denmark buys St Croix from } \\
\text { France }\end{array}$ & \\
\hline 1755 & Portugal & $\begin{array}{l}\text { Lisbon earthquake kills } \\
\text { 10,000-30,000, and shakes } \\
\text { confidence of Europe }\end{array}$ & \\
\hline 1755 & Middle East & $\begin{array}{l}\text { Earthquake in northern Persia } \\
\text { killing } 40,000\end{array}$ & \\
\hline 1755 & North America & $\begin{array}{l}\text { Acadians deported by British } \\
\text { from Nova Scotia }\end{array}$ & \\
\hline 1759 & Portugal & $\begin{array}{l}\text { Expulsion of Jesuits from } \\
\text { Portuguese territory }\end{array}$ & \\
\hline 1763 & North America & $\begin{array}{l}\text { Spain cedes Louisiana Territory } \\
\text { to France }\end{array}$ & \\
\hline 1766 & Europe & $\begin{array}{l}\text { King Charles III expels Jesuits } \\
\text { from Spanish Empire }\end{array}$ & $\begin{array}{l}\text { Jean-Jacques } \\
\text { Rousseau, } \\
\text { Confessions }\end{array}$ \\
\hline 1767 & Spanish Empire & $\begin{array}{l}\text { Expulsion of Jesuits from } \\
\text { Spanish territories }\end{array}$ & \\
\hline $1775-83$ & North America & $\begin{array}{l}\text { War of American } \\
\text { Independence }\end{array}$ & $\begin{array}{l}\text { Immanuel Kant, } \\
\text { Critique of Pure } \\
\text { Reason }\end{array}$ \\
\hline 1788 & France & & $\begin{array}{l}\text { Amis des Noirs } \\
\text { founded in France }\end{array}$ \\
\hline 1789 & France & French Revolution & $\begin{array}{l}\text { Declaration of the } \\
\text { Rights of Man } \\
\text { adopted by French } \\
\text { assembly }\end{array}$ \\
\hline 1789 & Europe & $\begin{array}{l}\text { Austrian Netherlands (Belgian } \\
\text { provinces) declare } \\
\text { independence from Vienna }\end{array}$ & \\
\hline 1789 & Britain & & $\begin{array}{l}\text { The Interesting } \\
\text { Narrative of the Life } \\
\text { of Olaudah Equiano, } \\
\text { or Gustava Vassa }\end{array}$ \\
\hline 1790 & Britain & & $\begin{array}{l}\text { Edmund Burke, } \\
\text { Reflections on the } \\
\text { Revolution in France }\end{array}$ \\
\hline 1791 & France & $\begin{array}{l}\text { French National Assembly } \\
\text { grants political rights to all } \\
\text { blacks and mulattoes born in } \\
\text { freedom, but not to slaves }\end{array}$ & \\
\hline $1791-1803$ & Caribbean & Haitian Revolution & \\
\hline 1792 & Denmark & $\begin{array}{l}\text { Danish ban on slave trade to } \\
\text { come into effect from } 1803\end{array}$ & \\
\hline 1793 & France & $\begin{array}{l}\text { The Reign of Terror } \\
\text { Execution of Louis XVI and } \\
\text { Marie Antoinette }\end{array}$ & \\
\hline
\end{tabular}




\begin{tabular}{|c|c|c|}
\hline Year & Regions & Historical Event \\
\hline & & $\begin{array}{l}\text { France declares war against } \\
\text { Britain }\end{array}$ \\
\hline 1794 & France & $\begin{array}{l}\text { Convention abolishes slavery } \\
\text { Fall of Robespierre }\end{array}$ \\
\hline $1795-1815$ & Africa & $\begin{array}{l}\text { Dutch lose control of Cape } \\
\text { Colony, S. Africa to British }\end{array}$ \\
\hline 1803 & North America & $\begin{array}{l}\text { The Louisiana Purchase: France } \\
\text { sells territory to US }\end{array}$ \\
\hline 1804 & Caribbean & Creation of Republic of Haiti \\
\hline 1804 & France & Napoleon crowned emperor \\
\hline 1805 & Europe & Battle of Trafalgar \\
\hline 1807 & Portugal/Brazil & $\begin{array}{l}\text { First Napoleonic invasion of } \\
\text { Portugal/ transfer of Portuguese } \\
\text { Royal Court to Brazil }\end{array}$ \\
\hline 1807 & Britain & $\begin{array}{l}\text { Abolition of slave trade in } \\
\text { British Empire }\end{array}$ \\
\hline 1811 & South America & Independence for Paraguay \\
\hline 1814 & Europe & $\begin{array}{l}\text { Kingdom of the Netherlands } \\
\text { created }\end{array}$ \\
\hline $1814-15$ & Europe & Congress of Vienna \\
\hline 1815 & South America & $\begin{array}{l}\text { Brazil attains status of kingdom } \\
\text { Independence for Uruguay } \\
\text { (ruled by Brazil until 1828) }\end{array}$ \\
\hline 1815 & Europe & Battle of Waterloo \\
\hline 1816 & South America & Independence for Argentina \\
\hline 1817 & France & France bans slave trade \\
\hline 1818 & South America & Independence for Chile \\
\hline 1820 & Europe & $\begin{array}{l}\text { Revolutions in Spain, } \\
\text { Portugal and Italy }\end{array}$ \\
\hline 1821 & C. America & Independence \\
\hline 1821 & Spain & Spain abolishes slave trade \\
\hline 1821 & Greece & Greek War of Independence \\
\hline 1822 & South America & Independence for Brazil \\
\hline 1823 & The Americas & Monroe Doctrine \\
\hline $1823-54$ & $\begin{array}{l}\text { South America/ } \\
\text { Caribbean }\end{array}$ & $\begin{array}{l}\text { Spanish American states } \\
\text { abolish slavery (Spanish } \\
\text { colonies: Puerto Rico 1872-3 } \\
\text { and Cuba 1886) }\end{array}$ \\
\hline 1825 & South America & $\begin{array}{l}\text { Simon Bolivar wins } \\
\text { independence for territories } \\
\text { now known as Venezuela, } \\
\text { Colombia, Panama } \\
\text { (until 1903), Ecuador, } \\
\text { Peru and Bolivia }\end{array}$ \\
\hline
\end{tabular}

\author{
Alexander von \\ Humboldt, Essai \\ politique sur l'île de \\ Cuba
}




\begin{tabular}{|c|c|c|}
\hline Year & Regions & Historical Event \\
\hline 1830 & North Africa & $\begin{array}{l}\text { Initial French occupation of } \\
\text { Algeria }\end{array}$ \\
\hline $1830-4$ & Africa & $\begin{array}{l}\text { Dutch-descended Boers make } \\
\text { 'Great Trek' into Natal }\end{array}$ \\
\hline 1832 & Cuba & \\
\hline 1833 & Britain & $\begin{array}{l}\text { Emancipation Act passed in } \\
\text { Britain }\end{array}$ \\
\hline 1836 & Sweden & Sweden abolishes slavery \\
\hline 1838 & Asia: Philippines & \\
\hline 1842 & Pacific & $\begin{array}{l}\text { Tahiti becomes French } \\
\text { protectorate }\end{array}$ \\
\hline 1845 & South America & \\
\hline 1846 & Sweden & Sweden abolishes slave trade \\
\hline $1846-8$ & Mexico/North America & Mexican-American War \\
\hline 1848 & Europe & $\begin{array}{l}\text { Revolutions all over Europe } \\
\text { Denmark abolishes slavery } \\
\text { Second abolition of } \\
\text { slavery in French Empire }\end{array}$ \\
\hline
\end{tabular}

Literary Event

Juan Francisco

Manzano,

Autobiografía

Francisco Baltazar,

Florante at Laura

Domingo Faustino

Sarmiento,

Facundo: civilisación

y barbarie

B. S. Ingemann,

Araberen $i$

Konstantinopel

Richard Burton, Goa, and the Blue

Mountains: Or, Six

Months of Sick

Leave

Gérard de Nerval, Le Voyage en

Orient

French arrive in New

Caledonia

Joseph Arthur

Comte de

Gobineau,

Essai sur l'inegalité

des races humaines

José Martiniano de

Alencar, O Guarany

Italian unification

Multatuli (Eduard

Douwes Dekker),

Max Havelaar 


\begin{tabular}{|c|c|c|c|}
\hline Year & Regions & Historical Event & Literary Event \\
\hline $1861-5$ & North America & American Civil War & \\
\hline $1861-7$ & Mexico & $\begin{array}{l}\text { French intervention in } \\
\text { Mexico }\end{array}$ & \\
\hline $1861-90$ & North America & Indian Wars & \\
\hline $1860 s$ & South America & & $\begin{array}{l}\text { Castro Alves } \\
\text { (Brazil) writes } \\
\text { abolitionist poems }\end{array}$ \\
\hline 1863 & Holland & Holland abolishes slave trade & \\
\hline 1864 & Europe & $\begin{array}{l}\text { Denmark loses Schleswig and } \\
\text { Holstein in war against Prussia } \\
\text { and Austria }\end{array}$ & \\
\hline $1864-70$ & South America & War of Triple Alliance & \\
\hline 1865 & Caribbean & Jamaica Rebellion & \\
\hline 1866 & Europe & $\begin{array}{l}\text { Austria defeated by Prussia } \\
\text { Prague Peace }\end{array}$ & \\
\hline 1867 & Europe & & $\begin{array}{l}\text { Karl Marx Das } \\
\text { Kapital }\end{array}$ \\
\hline 1867 & North America & $\begin{array}{l}\text { Knights of the White Camelia } \\
\text { formed in Louisiana }\end{array}$ & \\
\hline 1867-1918 & Germany & German Empire & \\
\hline 1868 & Middle East & Opening of Suez Canal & \\
\hline 1868 & Mexico & & $\begin{array}{l}\text { Ignacio Manuel } \\
\text { Altamirano, } \\
\text { Clemencia }\end{array}$ \\
\hline $1870-1$ & Europe & Franco-Prussian War & \\
\hline 1870-90 & South America & Unrest in Argentina & \\
\hline 1871 & Europe & Paris Commune & $\begin{array}{l}\text { Giuseppe Verdi, } \\
\text { Aida }\end{array}$ \\
\hline $1872-9$ & Argentina & & $\begin{array}{l}\text { José Hernández, } \\
\text { Martín Fierro }\end{array}$ \\
\hline 1872 & Africa & & $\begin{array}{l}\text { Henry Morton } \\
\text { Stanley, How I } \\
\text { Found Livingstone }\end{array}$ \\
\hline 1873 & Africa & $\begin{array}{l}\text { Zanzibar's public slave } \\
\text { markets close }\end{array}$ & \\
\hline $1873-4$ & Spain & First Spanish Republic & \\
\hline $1874-7$ & Africa & Stanley's journeys & \\
\hline $1879-83$ & South America & $\begin{array}{l}\text { War of Pacific between } \\
\text { Bolivia and Chile }\end{array}$ & \\
\hline 1880s-1914 & Africa & Scramble for Africa & \\
\hline 1881 & Brazil & & $\begin{array}{l}\text { Machado de Assis, } \\
\text { Memórias } \\
\text { Póstumas de Brás } \\
\text { Cubas }\end{array}$ \\
\hline $1881-8$ & Germany & & $\begin{array}{l}\text { Karl May, } \\
\text { Orientzyklus }\end{array}$ \\
\hline 1882 & Spanish Caribbean & & $\begin{array}{l}\text { Manuel de Jesus } \\
\text { Galván, Enriquillo }\end{array}$ \\
\hline
\end{tabular}




\begin{tabular}{|c|c|c|c|}
\hline Year & Regions & Historical Event & Literary Event \\
\hline $1882-1902$ & Middle East & $\begin{array}{l}\text { First mass Jewish migrations } \\
\text { to Palestine, largely from } \\
\text { East Europe }\end{array}$ & \\
\hline $1883-4$ & Africa & $\begin{array}{l}\text { German colonisation of south- } \\
\text { west Africa }\end{array}$ & \\
\hline $1884-5$ & Europe & Berlin Conference & \\
\hline 1885 & Africa & $\begin{array}{l}\text { German colonisation of parts of } \\
\text { East Africa (until 1917) } \\
\text { Congo private property of } \\
\text { Belgian king Leopold II } \\
\text { (until 1908) } \\
\text { 3-22 million Congolese killed } \\
\text { as a result of ruthless extraction } \\
\text { of natural resources }\end{array}$ & \\
\hline 1885 & Russia & $\begin{array}{l}\text { Nathan Birnbaum coins term } \\
\text { 'Zionism' }\end{array}$ & \\
\hline 1886 & Europe & $\begin{array}{l}\text { Slavery abolished in Spanish } \\
\text { colonies }\end{array}$ & $\begin{array}{l}\text { Gabriele } \\
\text { D'Annunzio, Isaotta } \\
\text { Guttadàuro ed altre } \\
\text { poesie }\end{array}$ \\
\hline 1887 & Europe & $\begin{array}{l}\text { Founding of World Zionist } \\
\text { Organization }\end{array}$ & \\
\hline 1887 & Asia/Europe & & $\begin{array}{l}\text { Jose Rizal, Noli Me } \\
\text { Tangere }\end{array}$ \\
\hline 1887 & Europe & & $\begin{array}{l}\text { Emilio Salgari, } L a \\
\text { Favorite del Mahdi }\end{array}$ \\
\hline 1888 & South America & Brazil abolishes slavery & \\
\hline 1889-97 & South America & Unrest in Brazil & \\
\hline 1889 & Philippines & & $\begin{array}{l}\text { Newspaper } L a \\
\text { Solidaridad starts } \\
\text { publication in } \\
\text { Manila }\end{array}$ \\
\hline 1889 & Mexico & & $\begin{array}{l}\text { Clorinda Matto de } \\
\text { Turner, Aves } \\
\text { sin nido }\end{array}$ \\
\hline 1890 & Africa/Europe & $\begin{array}{l}\text { Ultimatum clash between } \\
\text { British and Portuguese }\end{array}$ & \\
\hline 1890 & North America & Wounded Knee Massacre & \\
\hline 1891 & Philippines & & $\begin{array}{l}\text { Jose Rizal, El } \\
\text { Filibusterismo }\end{array}$ \\
\hline 1891 & Cuba & & $\begin{array}{l}\text { José Marti, Nuestra } \\
\text { América }\end{array}$ \\
\hline 1891 & Europe & & $\begin{array}{l}\text { Frieda von Bülow, } \\
\text { Der Konsul }\end{array}$ \\
\hline 1896 & Asia & $\begin{array}{l}\text { Spanish execute José Rizal for } \\
\text { promoting insurrection } \\
\text { in Philippines }\end{array}$ & \\
\hline
\end{tabular}




\begin{tabular}{|c|c|c|c|}
\hline Year & Regions & Historical Event & Literary Event \\
\hline 1896 & Europe/Africa & $\begin{array}{l}\text { Theodor Herzl, Zionist leader, } \\
\text { asserts need for a Jewish State } \\
\text { as a solution to European } \\
\text { anti-Semitism } \\
\text { Italy beaten in Abyssinia }\end{array}$ & $\begin{array}{l}\text { Frieda von Bülow, } \\
\text { Tropenkoller }\end{array}$ \\
\hline 1897 & Asia & $\begin{array}{l}\text { Kiachow (China) becomes a } \\
\text { German Protectorate } \\
\text { (until 1914) }\end{array}$ & \\
\hline 1897 & France & Dreyfus Affair & \\
\hline 1897 & Germany & & $\begin{array}{l}\text { Peter Altenberg, } \\
\text { Ashante }\end{array}$ \\
\hline 1898 & Caribbean/Asia & $\begin{array}{l}\text { Spanish-American War. } \\
\text { Spain loses her last colonies } \\
\text { in Americas }\end{array}$ & \\
\hline 1899 & Pacific & $\begin{array}{l}\text { German colonisation of } \\
\text { Western Samoa (until 1914) }\end{array}$ & \\
\hline 1899 & Asia & $\begin{array}{l}\text { Spanish cede Philippines } \\
\text { to USA }\end{array}$ & \\
\hline 1899-1902 & South America & $\begin{array}{l}\text { War of the Thousand Days in } \\
\text { Columbia }\end{array}$ & \\
\hline 1899-1902 & Africa & Boer War & \\
\hline 1900 & China & Boxer Rebellion & \\
\hline 1900 & Mexico & & $\begin{array}{l}\text { José Enrique Rodo, } \\
\text { Ariel }\end{array}$ \\
\hline 1901 & Middle East & & $\begin{array}{l}\text { Code of Hammurabi } \\
\text { discovered }\end{array}$ \\
\hline 1902 & Africa & $\begin{array}{l}\text { Benin becomes a French } \\
\text { colony (until 1960) }\end{array}$ & \\
\hline 1903 & Middle East & $\begin{array}{l}\text { Anglo-Palestine Bank } \\
\text { established as principal } \\
\text { financial institution of Jewish } \\
\text { community in Palestine }\end{array}$ & \\
\hline 1904 & Germany & & $\begin{array}{l}\text { Hanna Christaller, } \\
\text { Alfreds Frauen }\end{array}$ \\
\hline $1904-5$ & Asia & Russian-Japanese War & \\
\hline $1904-8$ & Africa & $\begin{array}{l}\text { Herero massacre in German } \\
\text { south-west Africa }\end{array}$ & \\
\hline 1904-14 & Middle East & $\begin{array}{l}\text { Second wave of Jewish migrants } \\
\text { from Russia and Poland to } \\
\text { Palestine }\end{array}$ & \\
\hline 1905 & North Europe & $\begin{array}{l}\text { Norway obtains independence } \\
\text { from Sweden after } 91 \text { years of } \\
\text { union }\end{array}$ & \\
\hline 1905 & USA & & $\begin{array}{l}\text { Mark Twain, King } \\
\text { Leopold's Soliloquy }\end{array}$ \\
\hline $1905-6$ & Africa & $\begin{array}{l}\text { Tangier Crisis (First Moroccan } \\
\text { Crisis) }\end{array}$ & \\
\hline
\end{tabular}




\begin{tabular}{lll} 
Year & Regions & Historical Event \\
\hline 1906 & Germany/Africa & \\
$1905-7$ & Africa & $\begin{array}{l}\text { Maji-Maji War in German } \\
\text { East Africa }\end{array}$ \\
$1905-9$ & Russia & $\begin{array}{l}\text { Pogroms killing estimated } \\
\text { 50,000 Jews }\end{array}$ \\
1907 & Africa & \\
& & \\
1907 & Germany & \\
& & \\
1908 & Africa/Europe & Congo to Belgian state \\
1909 & Middle East & Establishment of Tel Aviv as a \\
& Jewish city \\
$1910-24$ & Mexico & Mexican Revolution \\
1911 & Africa & Morocco divided between \\
& & French and Spanish \\
& & Agadir Crisis \\
& & Italy conquers Tripoli \\
& &
\end{tabular}

1911 Germany/Africa

1911-12 Europe

1912

1914

1914

1914

1915

1915-16

1915-17

1916

1916-18

Middle East

1917
Germany/Africa

Central America

Denmark

Uruguay

Mexico

Turkey

Europe

Europe

Middle East
Literary Event

Gustav Frenssen, Peter Moors Fahrt nach Südwest

Victor Segalen, Les Immémoriaux (pub. 1995)

Else Lasker-Schüler, Die Nächte Tino von Bagdads

Richard Küas, Vom Baum der Erkenntnis Gabriele

D'Annunzio, Le canzoni delle gesta d'oltremare Clara Brockmann, Briefe eines Mädchens aus Südwest

Opening of Panama Canal

Mathias Storch, singnagtugaK (A Greenlander's

Dream)

Delmira Agustini, El

Rosario de Eros

Mariano Azuela, Los de abajo

Allied defeat at Gallipoli Armenian genocide

Lenin, Imperialism, the Highest Stage of Capitalism (Russian)
Arab Revolt

Balfour Declaration by British promises Jews a homeland 


\begin{tabular}{|c|c|c|c|}
\hline Year & Regions & Historical Event & Literary Event \\
\hline & & $\begin{array}{l}\text { in Palestine } \\
\text { British conquer Baghdad and } \\
\text { Jerusalem }\end{array}$ & \\
\hline 1917 & Russia & $\begin{array}{l}\text { Russian Revolutions } \\
\text { End of Romanov Empire }\end{array}$ & \\
\hline 1917 & Denmark/Caribbean & $\begin{array}{l}\text { Danish West Indian colonies } \\
\text { sold to USA }\end{array}$ & \\
\hline 1918 & Europe & $\begin{array}{l}\text { First World War ends and } \\
\text { terminates German and } \\
\text { Austro-Hungarian Empires }\end{array}$ & \\
\hline 1918 & Peru & & $\begin{array}{l}\text { César Vallejo, } \\
\text { Heraldos negros }\end{array}$ \\
\hline 1919 & Europe & $\begin{array}{l}\text { Versailles Treaty (end of } \\
\text { German Empire) }\end{array}$ & $\begin{array}{l}\text { Oswald Spengler, } \\
\text { Untergang des } \\
\text { Abendlandes }\end{array}$ \\
\hline 1919 & South America & & $\begin{array}{l}\text { Alcides Arguedas, } \\
\text { Raza de bronce }\end{array}$ \\
\hline $1919-23$ & Middle East & $\begin{array}{l}\text { Third wave of mass Jewish } \\
\text { migrations to Palestine }\end{array}$ & \\
\hline $1919-26$ & North Africa & $\begin{array}{l}\text { Rif War: Spanish defeat Rif } \\
\text { Kabyles in northern } \\
\text { Morocco }\end{array}$ & \\
\hline $1919-33$ & Germany & Weimar Republic & \\
\hline 1920 & Germany & & $\begin{array}{l}\text { Erich Scheurmann, } \\
\text { Der Papalagi }\end{array}$ \\
\hline 1921 & Africa & & $\begin{array}{l}\text { Herman Grégoire, } \\
\text { Makako, singe } \\
\text { d'Afrique } \\
\text { René Maran, } \\
\text { Batouala, véritable } \\
\text { roman nègre }\end{array}$ \\
\hline 1921 & Germany & & $\begin{array}{l}\text { Willy Seidel, Der } \\
\text { Buschhahn }\end{array}$ \\
\hline 1921 & Middle East & Arab anti-Jewish riots & \\
\hline 1922 & Middle East & $\begin{array}{l}\text { Britain is given mandate for } \\
\text { Palestine by League of Nations }\end{array}$ & $\begin{array}{l}\text { T. E. Lawrence, } \\
\text { Seven Pillars of } \\
\text { Wisdom }\end{array}$ \\
\hline 1923 & Turkey & $\begin{array}{l}\text { End of Ottoman Empire; } \\
\text { proclamation of Turkish } \\
\text { Republic by Mustafa Kemal } \\
\text { Atatürk }\end{array}$ & \\
\hline $1923-30$ & Spain & $\begin{array}{l}\text { Primo de Rivera Spanish } \\
\text { dictator }\end{array}$ & \\
\hline $1924-9$ & Middle East & $\begin{array}{l}\text { Fourth wave of Jewish } \\
\text { migrations to Palestine }\end{array}$ & \\
\hline 1924 & South America & & $\begin{array}{l}\text { Luis Ambrosio } \\
\text { Morante, Tupac- } \\
\text { Amarú, drama en }\end{array}$ \\
\hline
\end{tabular}




\begin{tabular}{|c|c|c|c|}
\hline Year & Regions & Historical Event & Literary Event \\
\hline & & & $\begin{array}{l}\text { cinco actos, año de } \\
1821 \\
\text { Oswald de Andrade, } \\
\text { Pau-Brasil } \\
\text { Pablo Neruda, } \\
\text { poemas de amor y una } \\
\text { canción desesperada }\end{array}$ \\
\hline $1925-7$ & Central America & Nicaraguan Civil War & \\
\hline 1926 & Africa/Europe & & $\begin{array}{l}\text { Bakary Diallo, } \\
\text { Force-Bonté } \\
\text { Marie-Louis } \\
\text { Delhaise-Arnould, } \\
\text { Amedra: Roman de } \\
\text { moeurs nègres du } \\
\text { Congo belge }\end{array}$ \\
\hline 1926 & Europe & & $\begin{array}{l}\text { Hans Grimm, Volk } \\
\text { ohne Raum }\end{array}$ \\
\hline 1926 & South America & & $\begin{array}{l}\text { Ricardo Güiraldes, } \\
\text { Don Segundo Sombra } \\
\text { Peruvian journal: } \\
\text { Boletín Titicaca } \\
\text { (active to 1930) }\end{array}$ \\
\hline 1927 & Europe & & $\begin{array}{l}\text { Paul Morand, Magie } \\
\text { noire } \\
\text { Knud Rasmussen, } \\
\text { Across Arctic } \\
\text { America (5th Thule } \\
\text { Expedition) }\end{array}$ \\
\hline 1928 & South America & & $\begin{array}{l}\text { José Carlos } \\
\text { Mariátegui, Siete } \\
\text { ensayos de inter } \\
\text { pretación de la } \\
\text { realidad Peruana } \\
\text { Luis E. Valcárcel, } \\
\text { Tempestad en los } \\
\text { Andes }\end{array}$ \\
\hline 1928 & Turkey & $\begin{array}{l}\text { Turkey becomes secular: } \\
\text { Islam no longer in the } \\
\text { constitution as } \\
\text { state religion }\end{array}$ & $\begin{array}{l}\text { Halide Edib, The } \\
\text { Turkish Ordeal }\end{array}$ \\
\hline 1928 & Germany & Völkerschau, Stuttgart & \\
\hline 1928 & USA & & $\begin{array}{l}\text { Nella Larson, } \\
\text { Quicksand }\end{array}$ \\
\hline 1929 & USA & & $\begin{array}{l}\text { Nella Larson, } \\
\text { Passing }\end{array}$ \\
\hline 1929-39 & $\begin{array}{l}\text { Europe/Middle East/ } \\
\text { North America }\end{array}$ & $\begin{array}{l}\text { Fifth wave of Jewish migrations } \\
\text { to Palestine }\end{array}$ & \\
\hline 1929 & Middle East & Arab riots in Hebron & \\
\hline
\end{tabular}




\begin{tabular}{|c|c|c|c|}
\hline Year & Regions & Historical Event & Literary Event \\
\hline 1930 & Asia & Yen Bay rebellion, Vietnam & \\
\hline 1930 & Africa & & $\begin{array}{l}\text { Tshekisho Plaatje, } \\
\text { Mhudi (first novel } \\
\text { from an African } \\
\text { writer) }\end{array}$ \\
\hline 1930 & Europe & & $\begin{array}{l}\text { Willy Seidel, Der } \\
\text { Buschhahn, Ein } \\
\text { Roman aus der } \\
\text { Deutsch-Samoa }\end{array}$ \\
\hline 1930 & South America & & $\begin{array}{l}\text { Carlos Drummond } \\
\text { de Andrade (Brazil), } \\
\text { Alguma poesia } \\
\text { Uriel García (Peru), } \\
\text { El nuevo indio }\end{array}$ \\
\hline $1930 \mathrm{~s}$ & Europe/Caribbean & & $\begin{array}{l}\text { Négritude } \\
\text { movement in } \\
\text { France, North } \\
\text { Africa and the } \\
\text { Caribbean }\end{array}$ \\
\hline $1930-1$ & Belgium & & $\begin{array}{l}\text { Hergé, Tintin au } \\
\text { Congo }\end{array}$ \\
\hline $1930-7$ & Turkey & $\begin{array}{l}\text { Turkey suppresses Kurdish } \\
\text { revolts }\end{array}$ & \\
\hline $1930 s-74$ & Portugal & New State régime & \\
\hline 1931 & Africa & $\begin{array}{l}\text { Famine in Niger due to French } \\
\text { use of forced labour to build } \\
\text { roads and railways }\end{array}$ & \\
\hline 1931 & France & Colonial Exhibition, Paris & \\
\hline 1931 & Peru & & $\begin{array}{l}\text { César Vallejo, } \\
\text { Tungsteno }\end{array}$ \\
\hline $1931-9$ & Spain & Second Spanish Republic & \\
\hline 1932 & Africa & & $\begin{array}{l}\text { Henri Drum, Luéji } \\
\text { ya kondé }\end{array}$ \\
\hline 1932 & France & & $\begin{array}{l}\text { Céline, Voyage au } \\
\text { bout de la nuit }\end{array}$ \\
\hline 1933 & Japan/Europe & $\begin{array}{l}\text { Japan and Germany leave } \\
\text { League of Nations }\end{array}$ & \\
\hline 1933 & Germany & & $\begin{array}{l}\text { Franz Werfel, Die } \\
\text { vierzig Tage des } \\
\text { Musa Dagh }\end{array}$ \\
\hline 1934 & France & & $\begin{array}{l}\text { Michel Leiris, } \\
\text { L'Afrique fantôme }\end{array}$ \\
\hline 1934 & Portugal & & $\begin{array}{l}\text { Fernando Pessoa, } \\
\text { Message }\end{array}$ \\
\hline 1934 & South America & & $\begin{array}{l}\text { Jorge Icaza, } \\
\text { Huasipungo }\end{array}$ \\
\hline 1934 & Turkey & $\begin{array}{l}\text { Women get right to vote and } \\
\text { to abandon veil }\end{array}$ & \\
\hline
\end{tabular}




\begin{tabular}{|c|c|c|c|}
\hline Year & Regions & Historical Event & Literary Event \\
\hline 1935 & Europe & & $\begin{array}{l}\text { Clément Charoux, } \\
\text { Ameenah: Roman } \\
\text { mauricien } \\
\text { Hergé, L'Oreille } \\
\text { cassée } \\
\text { Pierre Ryckmans, } \\
\text { Allo! Congo! } \\
\text { Chroniques } \\
\text { Radiophoniques }\end{array}$ \\
\hline 1935 & Caribbean & & $\begin{array}{l}\text { Aimé Césaire uses } \\
\text { the term Négritude } \\
\text { in L'Étudiant noir }\end{array}$ \\
\hline 1935 & South America & & $\begin{array}{l}\text { Ciro Alegría (Peru), } \\
\text { La serpiente de oro } \\
\text { Víctor Raúl Haya de } \\
\text { la Torre, ¿A dónde va } \\
\text { Indoamérica? }\end{array}$ \\
\hline
\end{tabular}

1935-6 Africa

1936-9 Middle East

$1936 \quad$ Europe/Africa

1936-9

1936

1937

1937

1938

1938

1938

1939

1939

1939
Spain

Germany

Middle East

Italy

Denmark

Africa

Middle East

Turkey

Europe

Caribbean

Middle East
Italy conquers Ethiopia

(Abyssinia)

Arab anti-Jewish revolt in

Palestine

Spanish Civil War

Peel Commission recommends partition of Palestine into Jewish and Arab sections Italy leaves League of Nations

Woodhead Commission advises against partition recommended by Peel

Commission

Death of Atatürk

Italy attacks Albania
Jeanne Maquet-

Tombu, Le Siècle

marche

Adolf Hitler, Mein

Kampf

Karen Blixen Den

afrikanske farm

Paul Hazoumé,

Doguicimi
Jørgen-Frantz

Jacobsen, Barbara

Aimé Césaire,

Cahier d'un retour au

pays natal

Aharon Appelfeld, The Story of a Life 


\begin{tabular}{|c|c|c|c|}
\hline Year & Regions & Historical Event & Literary Event \\
\hline $1939-45$ & Europe & $\begin{array}{l}\text { Holocaust: mass extermination } \\
\text { of Jews by Nazis }\end{array}$ & \\
\hline 1939-78 & Spain & Franco régime & \\
\hline 1940 & Europe & Britain occupies Iceland & \\
\hline 1941 & Peru & & $\begin{array}{l}\text { Ciro Alegría, El } \\
\text { mundo es ancho y } \\
\text { ajeno }\end{array}$ \\
\hline 1942 & Germany & $\begin{array}{l}\text { Wannsee Conference planning } \\
\text { Endlösung der Judenfrage (Final } \\
\text { Solution to the Jewish Question) }\end{array}$ & \\
\hline 1942 & France & & $\begin{array}{l}\text { Albert Camus, } \\
\text { L'etranger (The } \\
\text { Stranger) }\end{array}$ \\
\hline 1944 & Caribbean & & $\begin{array}{l}\text { Jacques Roumain, } \\
\text { Gouverneurs de la } \\
\text { rosée }\end{array}$ \\
\hline 1944 & Iceland/Denmark & $\begin{array}{l}\text { Iceland cancels union with } \\
\text { Denmark and becomes } \\
\text { a republic }\end{array}$ & \\
\hline 1944 & Africa & $\begin{array}{l}\text { Free French forces and } \\
\text { representatives of France's } \\
\text { African colonies meet at } \\
\text { Brazzaville }\end{array}$ & \\
\hline 1944 & Denmark & & $\begin{array}{l}\text { Pâvia Petersen, } \\
\text { Niuvertorutsip pania, } \\
\text { (The Trading Station } \\
\text { Manager's Daughter) }\end{array}$ \\
\hline 1944 & South America & & $\begin{array}{l}\text { Jorge Luis Borges, } \\
\text { Ficciones }\end{array}$ \\
\hline 1945 & Africa/France & & $\begin{array}{l}\text { Léopold Sédar } \\
\text { Senghor, Chants } \\
\text { d'ombre }\end{array}$ \\
\hline 1946 & French Empire & $\begin{array}{l}\text { Creation of the Union } \\
\text { Française, and } \\
\text { 'departmentalisation' of } \\
\text { former colonies }\end{array}$ & \\
\hline 1946 & Africa & & $\begin{array}{l}\text { Egide Straven, } \\
\text { Kapiri-Pi. Roman } \\
\text { africain }\end{array}$ \\
\hline 1946 & Faeroe Islands/Denmark & $\begin{array}{l}\text { Faeroe Islands in a shock } \\
\text { referendum votes in favour of } \\
\text { independence from Denmark }\end{array}$ & \\
\hline 1946 & Poland & $\begin{array}{l}\text { Pogrom of Polish Jews surviving } \\
\text { Holocaust at Kielche, } 14 \text { July }\end{array}$ & \\
\hline 1947 & Middle East & $\begin{array}{l}\text { UN General Assembly } \\
\text { approves a partition plan for } \\
\text { Palestine that is accepted by } \\
\text { Jews but rejected by Arabs }\end{array}$ & \\
\hline
\end{tabular}




\begin{tabular}{lll} 
Year & Regions & Historical Event \\
\hline 1947 & Africa & French massacres in \\
& Madagascar. \\
& Estimates of number of victims \\
& vary from a few thousands to \\
& more than 300,000 \\
& &
\end{tabular}

$1948 \quad$ Europe

Universal Declaration of Human Rights

Literary Event

Pierre Ryckmans, Barabara

Paul LomamiTshibamba, Ngando (Le crocodile) Anthologie de la nouvelle poésie nègre et malgache, ed. Léopold Sédar Senghor Jean-Paul Sartre, 'Orphée Noir'

Middle East

State of Israel established, followed by war with Egypt, Syria, Jordan, Lebanon and Iraq

Faeroe Islands granted home rule

Africa

Massacres in Cote d'Ivoire killing 100,000

Apartheid laws passed in South Africa

Ernesto Sábato, El

Túnel

Fernando de Castro

Soromenho, Terra

morta

1949 Caribbean

$1949 \quad$ Portugal

1949 Middle East

Jerusalem is divided between

Alejo Carpentier, El reino de este mundo José Régio, El-Rei

Sebastião: Teatro Israel and Jordan; mass immigrations to Israel

1950 Caribbean

$1950 \quad$ France

Middle East

Persia nationalises oil industry

Miguel Ángel, Hombres de maiz Joseph Zobel, La Rue cases-nègres Marguerite Duras, Un Barrage contre le Pacifique

1952 Europe

Africa

Africa

Mau Mau Uprising in Kenya

Frantz Fanon, Peau noire, masques blancs

Camara Laye (Guinea), L'Enfant noir (French)

Greenland changes from colony to Danish county 


\begin{tabular}{|c|c|c|c|}
\hline Year & Regions & Historical Event & Literary Event \\
\hline 1954 & Africa & & $\begin{array}{l}\text { Henri Cornélus, } \\
\text { Kufa }\end{array}$ \\
\hline 1954 & Asia & $\begin{array}{l}\text { French defeated at } \\
\text { Dien Bien Phu }\end{array}$ & \\
\hline 1955 & Africa/USA & & $\begin{array}{l}\text { Robert Ruark, } \\
\text { Something of Value }\end{array}$ \\
\hline 1955 & Caribbean & & $\begin{array}{l}\text { Aimé Césaire, } \\
\text { Discours sur le } \\
\text { colonialisme }\end{array}$ \\
\hline 1955 & Turkey & & $\begin{array}{l}\text { Yaşal Kemal, } \\
\text { Memed, My Hawk }\end{array}$ \\
\hline 1956 & Middle East & $\begin{array}{l}\text { Egypt nationalises Suez Canal } \\
\text { Israel invades Sinai }\end{array}$ & \\
\hline 1956 & Africa & $\begin{array}{l}\text { Independence for Tunisia and } \\
\text { Morocco }\end{array}$ & $\begin{array}{l}\text { Kateb Yacine, } \\
\text { Nedjma }\end{array}$ \\
\hline 1956 & Europe & $\begin{array}{l}\text { Soviet Union crushes uprisings } \\
\text { in Poland and Hungary }\end{array}$ & \\
\hline 1956 & Brazil & & $\begin{array}{l}\text { João Guimarães } \\
\text { Rosa, Grande Sertão: } \\
\text { Veredas }\end{array}$ \\
\hline 1957 & Africa & Independence for Ghana & $\begin{array}{l}\text { Ousmane Sembène, } \\
\text { Le Docker noir }\end{array}$ \\
\hline 1957 & Caribbean & & $\begin{array}{l}\text { Aimé Césaire, Et les } \\
\text { chiens se taisaient }\end{array}$ \\
\hline 1957 & Middle East & $\begin{array}{l}\text { Israel withdraws from the } \\
\text { Gaza Strip }\end{array}$ & \\
\hline 1957 & South America & & $\begin{array}{l}\text { Gamaliel Churata, } \\
\text { El Pez de Oro }\end{array}$ \\
\hline 1958 & Africa & & $\begin{array}{l}\text { Chinua Achebe, } \\
\text { Things Fall Apart }\end{array}$ \\
\hline 1958 & Africa & $\begin{array}{l}\text { Afrikaner-ruled South Africa } \\
\text { becomes independent of } \\
\text { British rule }\end{array}$ & \\
\hline 1959 & Africa & & $\begin{array}{l}\text { Bernard Dadié, Un } \\
\text { Nègre à Paris } \\
\text { Frantz Fanon, L'An } \\
\text { V de la révolution } \\
\text { algérienne } \\
\text { Marcel Tinel, Le } \\
\text { Monde de Nzakomba }\end{array}$ \\
\hline 1959 & Europe & & $\begin{array}{l}\text { André Schwarz- } \\
\text { Bart, Le Dernier des } \\
\text { Justes }\end{array}$ \\
\hline 1959 & Mexico & & $\begin{array}{l}\text { Carlos Fuentes, } L a \\
\text { región más } \\
\text { transparente }\end{array}$ \\
\hline
\end{tabular}




\begin{tabular}{|c|c|c|c|}
\hline Year & Regions & Historical Event & Literary Event \\
\hline 1960 & Africa & $\begin{array}{l}\text { Zaire (formerly the Belgian } \\
\text { Congo) becomes independent } \\
\text { from Belgium }\end{array}$ & $\begin{array}{l}\text { Ousmane Sembène, } \\
\text { Les Bout de bois } \\
\text { de dieu }\end{array}$ \\
\hline 1960 & Europe/Africa & & $\begin{array}{l}\text { Daniel Gillès, } L a \\
\text { Termitière }\end{array}$ \\
\hline 1960 & South America & & $\begin{array}{l}\text { Clarice Lispector, } \\
\text { Laços de família }\end{array}$ \\
\hline 1960 & Europe/Africa & & $\begin{array}{l}\text { Joseph Esser, Matuli, } \\
\text { fille d'Afrique }\end{array}$ \\
\hline 1960 & Turkey & $\begin{array}{l}\text { Military coup overthrows } \\
\text { democratic government }\end{array}$ & \\
\hline 1961 & Africa & & $\begin{array}{l}\text { António Agostinho } \\
\text { Neto, Poemas }\end{array}$ \\
\hline 1961 & Asia & & $\begin{array}{l}\text { Orlando da Costa, } \\
\text { O Signo da Ira }\end{array}$ \\
\hline 1961 & Portugal/India/Africa & $\begin{array}{l}\text { Uprisings against Portuguese } \\
\text { colonialism in Angola } \\
\text { Goa ceases to be a Portuguese } \\
\text { colony }\end{array}$ & \\
\hline 1961 & Europe & & $\begin{array}{l}\text { F. Fanon, Les } \\
\text { Damnés de la terre }\end{array}$ \\
\hline 1961 & Germany & Berlin Wall erected & \\
\hline 1961 & Africa & & $\begin{array}{l}\text { Cheikh Hamidou } \\
\text { Kane, L'Aventure } \\
\text { ambiguë }\end{array}$ \\
\hline 1961 & Caribbean & Bay of Pigs & \\
\hline 1961 & France & $\begin{array}{l}\text { Massacre in Paris of pro- } \\
\text { Algerian demonstrators, } \\
17 \text { October }\end{array}$ & \\
\hline 1961 & South America & & $\begin{array}{l}\text { José María } \\
\text { Arguedas, Los Ríos } \\
\text { Profundos }\end{array}$ \\
\hline 1962 & Africa & $\begin{array}{l}\text { Evian Accords: Algeria } \\
\text { becomes independent } \\
\text { from French rule }\end{array}$ & $\begin{array}{l}\text { Assia Djebar, Les } \\
\text { Enfants du nouveau } \\
\text { monde }\end{array}$ \\
\hline 1962 & Caribbean & Cuban Missile Crisis & \\
\hline 1963 & Africa & $\begin{array}{l}\text { Kenya becomes independent } \\
\text { from British rule }\end{array}$ & $\begin{array}{l}\text { Jean Ikelle-Matiba, } \\
\text { Cette Afrique-la! }\end{array}$ \\
\hline 1964 & Africa & & $\begin{array}{l}\text { José Luandino } \\
\text { Vieira, Luuanda: } \\
\text { Short Stories of } \\
\text { Angola (Portuguese) }\end{array}$ \\
\hline 1964 & Caribbean & & $\begin{array}{l}\text { Edouard Glissant, Le } \\
\text { quatrième siècle }\end{array}$ \\
\hline 1964 & Middle East & $\begin{array}{l}\text { The Palestine Liberation } \\
\text { Organization established by } \\
\text { the Arab League }\end{array}$ & \\
\hline
\end{tabular}




\begin{tabular}{|c|c|c|c|}
\hline Year & Regions & Historical Event & Literary Event \\
\hline 1965 & Africa & Rhodesia declares independence & \\
\hline $1965-73$ & Asia & Vietnam War & \\
\hline 1965 & Africa & & $\begin{array}{l}\text { James Ngugi, The } \\
\text { River Between } \\
\text { Thomas Kanza, Sans } \\
\text { rancune }\end{array}$ \\
\hline 1966 & Africa & & $\begin{array}{l}\text { Albert Memmi, La } \\
\text { Libération du Juif } \\
\text { Flora Nwapa, Efuru } \\
\text { (first novel by a } \\
\text { woman writer from } \\
\text { Africa) }\end{array}$ \\
\hline 1966 & Middle East & & $\begin{array}{l}\text { Aziz Nesin, Istanbul } \\
\text { Boy (Turkish) } \\
\text { S.Y. Agnon is } \\
\text { declared joint } \\
\text { winner of the Nobel } \\
\text { Prize for Literature }\end{array}$ \\
\hline 1966 & South America & & $\begin{array}{l}\text { Arturo Borda, El } \\
\text { loco } \\
\text { Carolina María de } \\
\text { Jesús, Child of the } \\
\text { Dark (Portuguese) } \\
\text { Mario Vargas Llosa, } \\
\text { La Casa Verde }\end{array}$ \\
\hline 1967 & Africa & & $\begin{array}{l}\text { Aimé Césaire, Une } \\
\text { saison au Congo } \\
\text { (French) }\end{array}$ \\
\hline 1967 & Denmark & & $\begin{array}{l}\text { Thorkild Hansen, } \\
\text { Slavernes kyst }\end{array}$ \\
\hline 1967 & Middle East & $\begin{array}{l}\text { The Six-Day War: Israel wrests } \\
\text { control of several Arab- } \\
\text { occupied regions }\end{array}$ & \\
\hline 1967 & South America & & $\begin{array}{l}\text { Gabriel García } \\
\text { Márquez, Cien años } \\
\text { de soledad }\end{array}$ \\
\hline 1968 & Caribbean & & $\begin{array}{l}\text { Aimé Césaire, Une } \\
\text { tempête }\end{array}$ \\
\hline 1968 & Africa & $\begin{array}{l}\text { Independence for Equatorial } \\
\text { Guinea (from Spain) }\end{array}$ & $\begin{array}{l}\text { Ahmadou } \\
\text { Kourouma, Les } \\
\text { Soleils des } \\
\text { indépendances } \\
\text { Malian Yambo } \\
\text { Ouologuem, Le } \\
\text { Devoir de violence } \\
\text { Kwame Nkrumah, } \\
\text { The Beautiful Ones } \\
\text { Are Not Yet Born }\end{array}$ \\
\hline
\end{tabular}




\begin{tabular}{|c|c|c|c|}
\hline Year & Regions & Historical Event & Literary Event \\
\hline 1968 & Caribbean & & $\begin{array}{l}\text { Marie Chauvet, } \\
\text { Amour, colère, folie }\end{array}$ \\
\hline 1968 & Europe & & $\begin{array}{l}\text { Conor Cruise } \\
\text { O'Brien, Murderous } \\
\text { Angels } \\
\text { Thorkild Hansen, } \\
\text { Slavernes skibe } \\
\text { (Ships of Slaves) }\end{array}$ \\
\hline 1969 & Africa & & $\begin{array}{l}\text { Luís Bernardo } \\
\text { Honwana, We Killed } \\
\text { Mangy-Dog and } \\
\text { Other Mozambique } \\
\text { Stories (Portuguese) }\end{array}$ \\
\hline 1969 & Cuba & & $\begin{array}{l}\text { Heberto Padilla, } \\
\text { Fuera del juego }\end{array}$ \\
\hline 1969 & Peru & & $\begin{array}{l}\text { Mario Vargas Llosa, } \\
\text { Conversación en } \\
\text { la Catedral }\end{array}$ \\
\hline 1970s & Africa & $\begin{array}{l}\text { Angola and Mozambique } \\
\text { become independent from } \\
\text { Portuguese rule }\end{array}$ & \\
\hline 1970 & Denmark & & $\begin{array}{l}\text { Thorkild Hansen, } \\
\text { Slavernes øer } \\
\text { (Islands of Slaves) }\end{array}$ \\
\hline 1971 & Africa & & $\begin{array}{l}\text { Kofi Awonoor, This } \\
\text { Earth, My Brother }\end{array}$ \\
\hline 1971 & Cuba & & $\begin{array}{l}\text { Roberto Fernández } \\
\text { Retamar, 'Calibán' }\end{array}$ \\
\hline 1971 & Europe & & $\begin{array}{l}\text { Antonio Gramsci, } \\
\text { Selections from the } \\
\text { Prison Notebooks }\end{array}$ \\
\hline 1971 & South America & & $\begin{array}{l}\text { José María } \\
\text { Arguedas, El zorro de } \\
\text { arriba y el zorro de } \\
\text { abajo } \\
\text { José Watanabe, } \\
\text { Album de Familia }\end{array}$ \\
\hline 1972 & Caribbean & & $\begin{array}{l}\text { Simone Schwarz- } \\
\text { Bart, Pluie et vent } \\
\text { sur Télumée Miracle }\end{array}$ \\
\hline 1972 & Europe & & $\begin{array}{l}\text { André Schwarz- } \\
\text { Bart, La Mulâtresse } \\
\text { solitude }\end{array}$ \\
\hline 1973 & Middle East & Egypt and Syria attack Israel & \\
\hline 1973 & South America & Allende's fall in Chile & \\
\hline 1974 & Africa & & $\begin{array}{l}\text { António Agostinho } \\
\text { Neto, Sagrada } \\
\text { Esperança }\end{array}$ \\
\hline
\end{tabular}




\begin{tabular}{|c|c|c|c|}
\hline Year & Regions & Historical Event & Literary Event \\
\hline 1974 & Portugal & $\begin{array}{l}\text { Carnation Revolution in } \\
\text { Portugal ends nearly five } \\
\text { decades of dictatorship }\end{array}$ & \\
\hline 1974 & Paraguay & & $\begin{array}{l}\text { Augusto Roa Bastos, } \\
\text { Yo el Supremo }\end{array}$ \\
\hline 1975 & Africa & $\begin{array}{l}\text { Angola, Cape Verde, São } \\
\text { Tomé e Príncipe, Guinea } \\
\text { Bissau and Mozambique gain } \\
\text { independence from } \\
\text { Portugal }\end{array}$ & \\
\hline 1975 & Mexico & & $\begin{array}{l}\text { Carlos Fuentes, } \\
\text { Terra Nostra }\end{array}$ \\
\hline 1975 & Middle East & $\begin{array}{l}\text { UN passes resolution equating } \\
\text { Zionism with racism }\end{array}$ & \\
\hline 1976 & Africa & & $\begin{array}{l}\text { Cheikh Anta Diop, } \\
\text { The African Origin } \\
\text { of Civilization } \\
\text { (French) }\end{array}$ \\
\hline 1976 & Asia & & $\begin{array}{l}\text { Francisco Borja da } \\
\text { Costa, } \\
\text { Revolutionary Poems } \\
\text { in the Struggle } \\
\text { Against Colonialism: } \\
\text { Timorese } \\
\text { Nationalist Verse }\end{array}$ \\
\hline 1976 & South America & & $\begin{array}{l}\text { Manuel Puig, El beso } \\
\text { de la mujer araña }\end{array}$ \\
\hline 1977 & Africa & & $\begin{array}{l}\text { Grégoire Pessaret, } \\
\text { Émile et le destin }\end{array}$ \\
\hline 1978 & Central America & $\begin{array}{l}\text { Sandinista Revolt in } \\
\text { Nicaragua }\end{array}$ & \\
\hline 1978 & Europe & & $\begin{array}{l}\text { Ivan Reisdorff, } \\
\text { L'Homme qui } \\
\text { demanda du feu } \\
\text { Uwe Timm, } \\
\text { Morenga }\end{array}$ \\
\hline 1979 & Africa & & $\begin{array}{l}\text { Mariama Bâ, Une si } \\
\text { longue lettre } \\
\text { Sony Labou Tansi, } \\
\text { La Vie et demie } \\
\text { Valentin Yves } \\
\text { Mudimbe, L'Écart } \\
\text { Williams Sassine, Le } \\
\text { Jeune homme de sable }\end{array}$ \\
\hline 1979 & Portugal/Africa & & $\begin{array}{l}\text { António Lobo } \\
\text { Antunes, South of } \\
\text { Nowhere } \\
\text { (Portuguese) }\end{array}$ \\
\hline
\end{tabular}




\begin{tabular}{|c|c|c|c|}
\hline Year & Regions & Historical Event & Literary Event \\
\hline 1979 & Caribbean & & $\begin{array}{l}\text { César Leante, Los } \\
\text { guerrilleros negros } \\
\text { René Depestre, Le } \\
\text { Mât de cocagne }\end{array}$ \\
\hline 1979 & Middle East & $\begin{array}{l}\text { Israel signs peace treaty with } \\
\text { Israel } \\
\text { Islamic Revolution in Iran } \\
\text { USSR invades Afghanistan }\end{array}$ & \\
\hline 1979 & Greenland & Greenland achieves home rule & \\
\hline 1980 & Africa & & Pepetela, Mayombe \\
\hline 1980 & Caribbean & & $\begin{array}{l}\text { Axel Gauvin, } \\
\text { Quartier trois lettres }\end{array}$ \\
\hline 1980 & Portugal & & $\begin{array}{l}\text { Alexandre } \\
\text { Herculano, Eurico, o } \\
\text { Presbitero }\end{array}$ \\
\hline 1981 & Africa & & $\begin{array}{l}\text { Nadine Nyangoma, } \\
\text { Le Chant des fusillés } \\
\text { Paul Lomami- } \\
\text { Tshibamba, } \\
\text { Ngemena }\end{array}$ \\
\hline 1981 & Caribbean & & $\begin{array}{l}\text { Edouard Glissant, Le } \\
\text { Discours antillais }\end{array}$ \\
\hline 1982 & Africa & & $\begin{array}{l}\text { Emmanuel Dongala, } \\
\text { Jazz et vin de palme }\end{array}$ \\
\hline 1982 & Europe & & $\begin{array}{l}\text { Fernando Pessoa, } \\
\text { The Book of } \\
\text { Disquiet Composed } \\
\text { by Bernardo Soares } \\
\text { Teolinda Gersão, } \\
\text { Paisagem Com } \\
\text { Mulher e Mar ao } \\
\text { Fundo }\end{array}$ \\
\hline 1982 & Middle East & $\begin{array}{l}\text { Israel launches attack in } \\
\text { southern Lebanon; withdraws } \\
\text { from most parts by } 1983\end{array}$ & $\begin{array}{l}\text { Amos } \mathrm{Oz} \text {, In the } \\
\text { Land of Israel } \\
\text { (Hebrew) }\end{array}$ \\
\hline 1982 & Chile & & $\begin{array}{l}\text { Isabel Allende, } L a \\
\text { casa de los espíritus }\end{array}$ \\
\hline 1983 & Africa & $\begin{array}{l}\text { Mauritania abolishes } \\
\text { slavery - the last country } \\
\text { in the world to do so }\end{array}$ & $\begin{array}{l}\text { Mehdi Charef, Le } \\
\text { Thé au harem } \\
\text { d'Archi Ahmed } \\
\text { Michèle Rakotoson, } \\
\text { Le Bain des reliques: } \\
\text { Roman malgache } \\
\text { (pub. 1988) } \\
\text { Omer Marchal, } \\
\text { Afrique,Afrique } \\
\text { Tété-Michel } \\
\text { Kpomassie, }\end{array}$ \\
\hline
\end{tabular}




\begin{tabular}{|c|c|c|c|}
\hline Year & Regions & Historical Event & Literary Event \\
\hline \multirow{6}{*}{1983} & \multirow{6}{*}{ Central America } & \multirow{6}{*}{ US invasion of Grenada } & L'Africain du \\
\hline & & & Grœenland \\
\hline & & & Rigoberta Menchú, \\
\hline & & & I, Rigoberta \\
\hline & & & Menchu: An Indian \\
\hline & & & Woman in Guatemala \\
\hline \multirow[t]{3}{*}{1984} & \multirow[t]{3}{*}{ Africa } & & Pepetela, Yaka \\
\hline & & & Touati Fettouma, \\
\hline & & & Printemps désespéré \\
\hline \multirow[t]{3}{*}{1984} & \multirow[t]{3}{*}{ France } & & Didier Daeninckx, \\
\hline & & & Meurtres pour \\
\hline & & & mémoire \\
\hline \multirow[t]{2}{*}{1984} & \multirow{2}{*}{\multicolumn{2}{|c|}{ North America }} & J. Poulin, \\
\hline & & & Volkswagen Blues \\
\hline \multirow[t]{2}{*}{1984} & \multirow{2}{*}{\multicolumn{2}{|c|}{ South America }} & Julio Cortázar, \\
\hline & & & $\begin{array}{l}\text { Nicaragua tan violen } \\
\text { tamente dulce }\end{array}$ \\
\hline \multirow[t]{4}{*}{1985} & \multirow[t]{4}{*}{ Africa } & & Assia Djebar, \\
\hline & & & L'Amour, la fantasia \\
\hline & & & Tahar Ben Jelloun, \\
\hline & & & L'Enfant de sable \\
\hline \multirow[t]{4}{*}{1985} & \multirow{3}{*}{\multicolumn{2}{|c|}{ Caribbean }} & Dany Laferrière, \\
\hline & & & Comment faire \\
\hline & & & l'amour avec un nègre \\
\hline & & & sans se fatiguer \\
\hline \multirow[t]{2}{*}{1985} & \multicolumn{2}{|l|}{ Europe } & Jacques de Decker, \\
\hline & & & La Grande roué \\
\hline \multirow[t]{2}{*}{1986} & \multirow{2}{*}{\multicolumn{2}{|c|}{ Africa }} & Mpoyi Buatu, La re- \\
\hline & & & Production \\
\hline \multirow[t]{3}{*}{1986} & \multirow{3}{*}{\multicolumn{2}{|c|}{ Caribbean }} & Maryse Condé, Moi, \\
\hline & & & Tituba, sorcière \\
\hline & & & noire de Salem \\
\hline \multirow[t]{7}{*}{1986} & \multirow[t]{7}{*}{ Portugal } & & José Saramago, The \\
\hline & & & Stone Raft \\
\hline & & & (Portuguese) \\
\hline & & & José Saramago, The \\
\hline & & & Year of the Death \\
\hline & & & of Ricardo Reis \\
\hline & & & (Portuguese) \\
\hline \multirow[t]{8}{*}{1987} & Africa & & Calixthe Beyala, \\
\hline & & & C'est le soleil qui \\
\hline & & & m'a brûlée \\
\hline & & & Chinua Achebe, \\
\hline & & & Anthills of the \\
\hline & & & Savannah \\
\hline & & & Tahar Ben Jelloun, \\
\hline & & & La Nuit sacrée \\
\hline
\end{tabular}




\begin{tabular}{lll} 
Year & Regions & Historical Event \\
\hline 1987 & Caribbean & \\
1987 & Germany & \\
1987 & Mexico/USA & \\
& & \\
1987 & Middle East & Violent protests by Palestinians \\
& & in the West Bank and \\
& Gaza Strip
\end{tabular}
your Life: Peasant Tales of Tragedy in Mozambique (Portuguese) U. Xitu, The World of 'Mestre' Tamoda Anne Hébert, Le Premier Jardin

Middle East

Independent state of Palestine proclaimed by the Palestinian National Council

Pacific

The Matignon Accords grant considerable autonomy to New Caledonia

Africa

US Panama campaign

António Lobo Antunes, The Return of the Caravels: A Novel (Portuguese) Lídia Jorge, A Costa dos Murmúrios Azouz Begag, Béni ou le paradis privé Manuel Alegre, Jornada de África: Romance de Amor e Morte do Alferes Sebastião Maryse Condé, Traversée de la mangrove

José de Sousa Saramago, História 


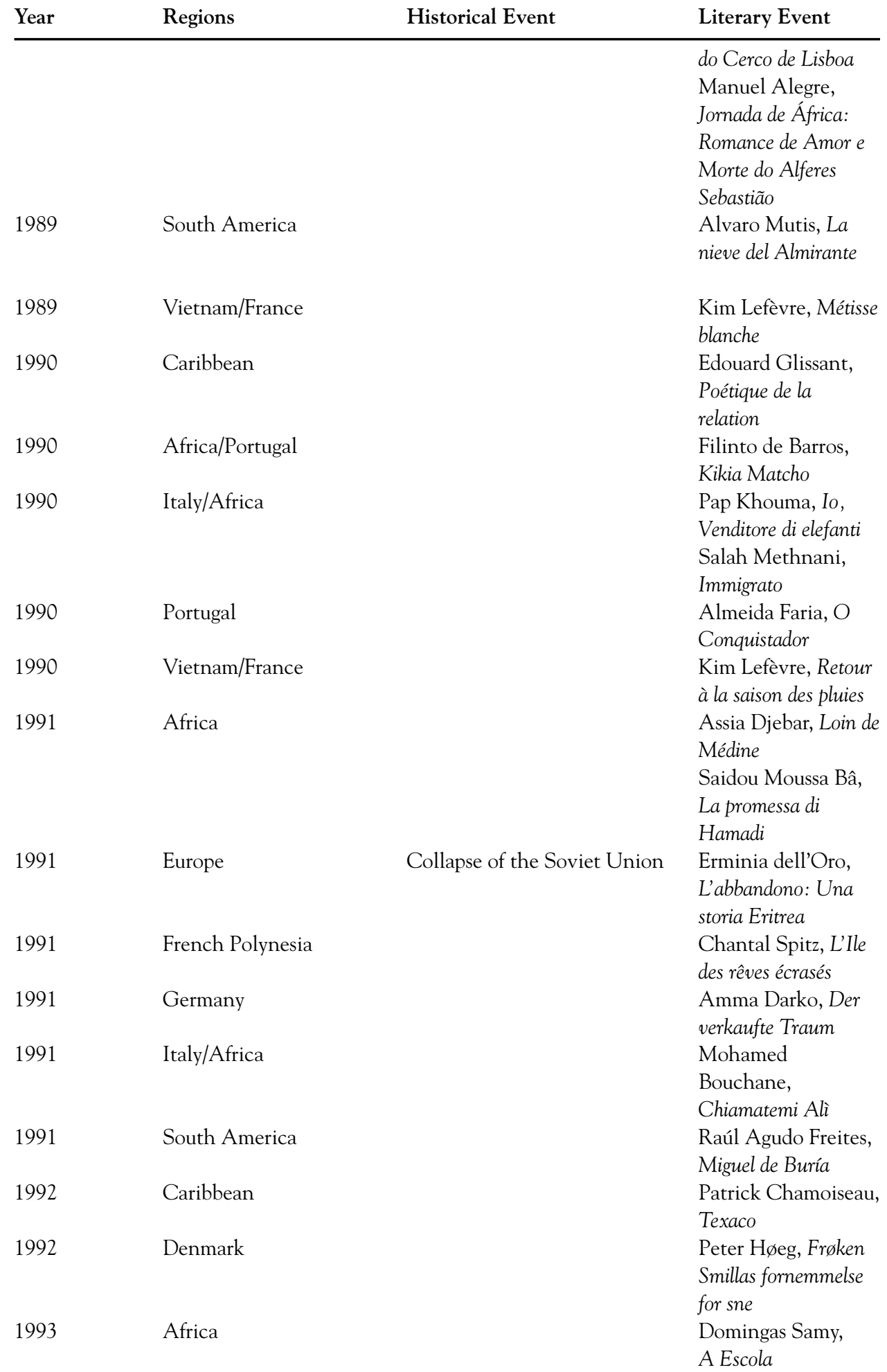




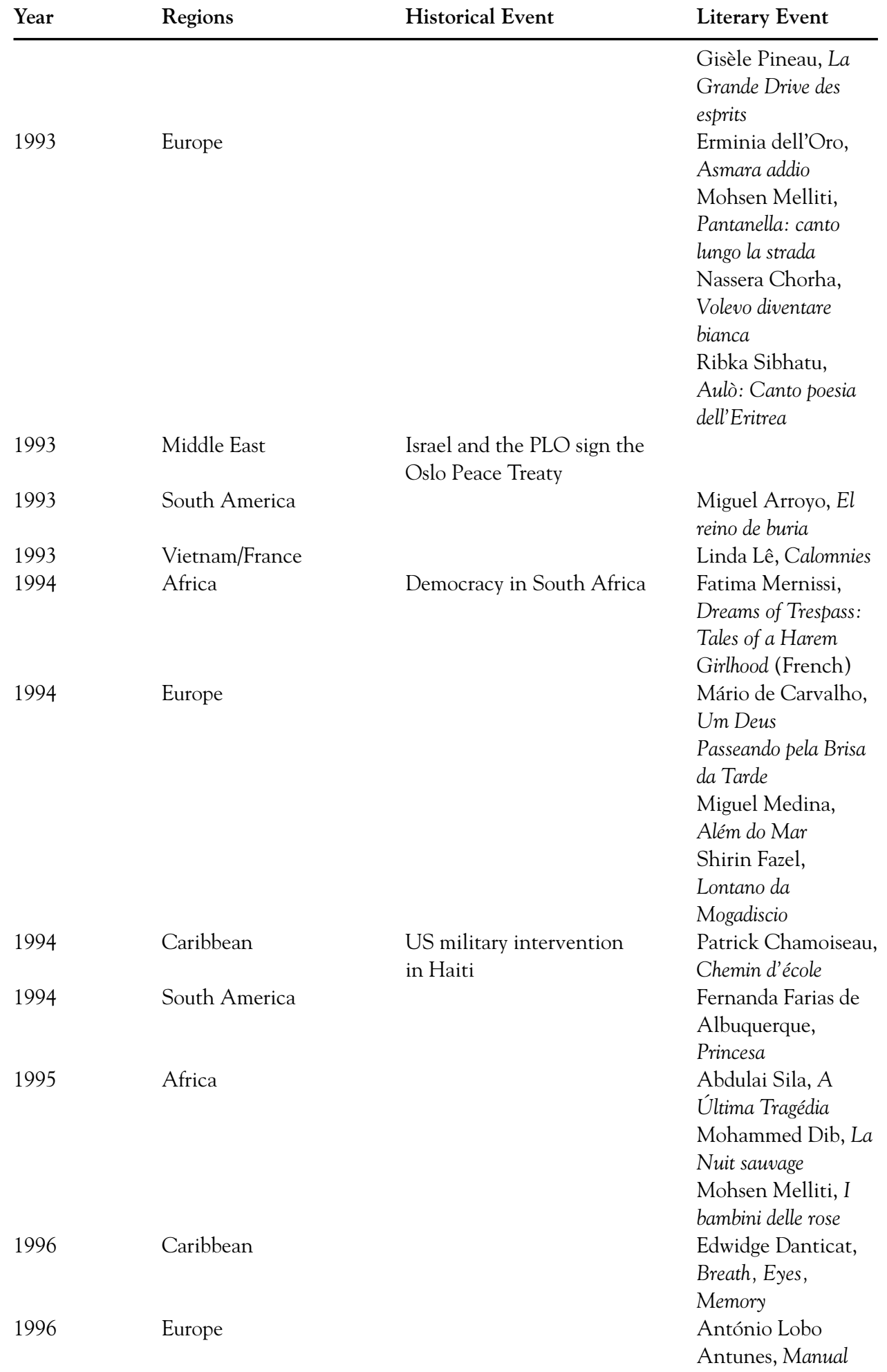




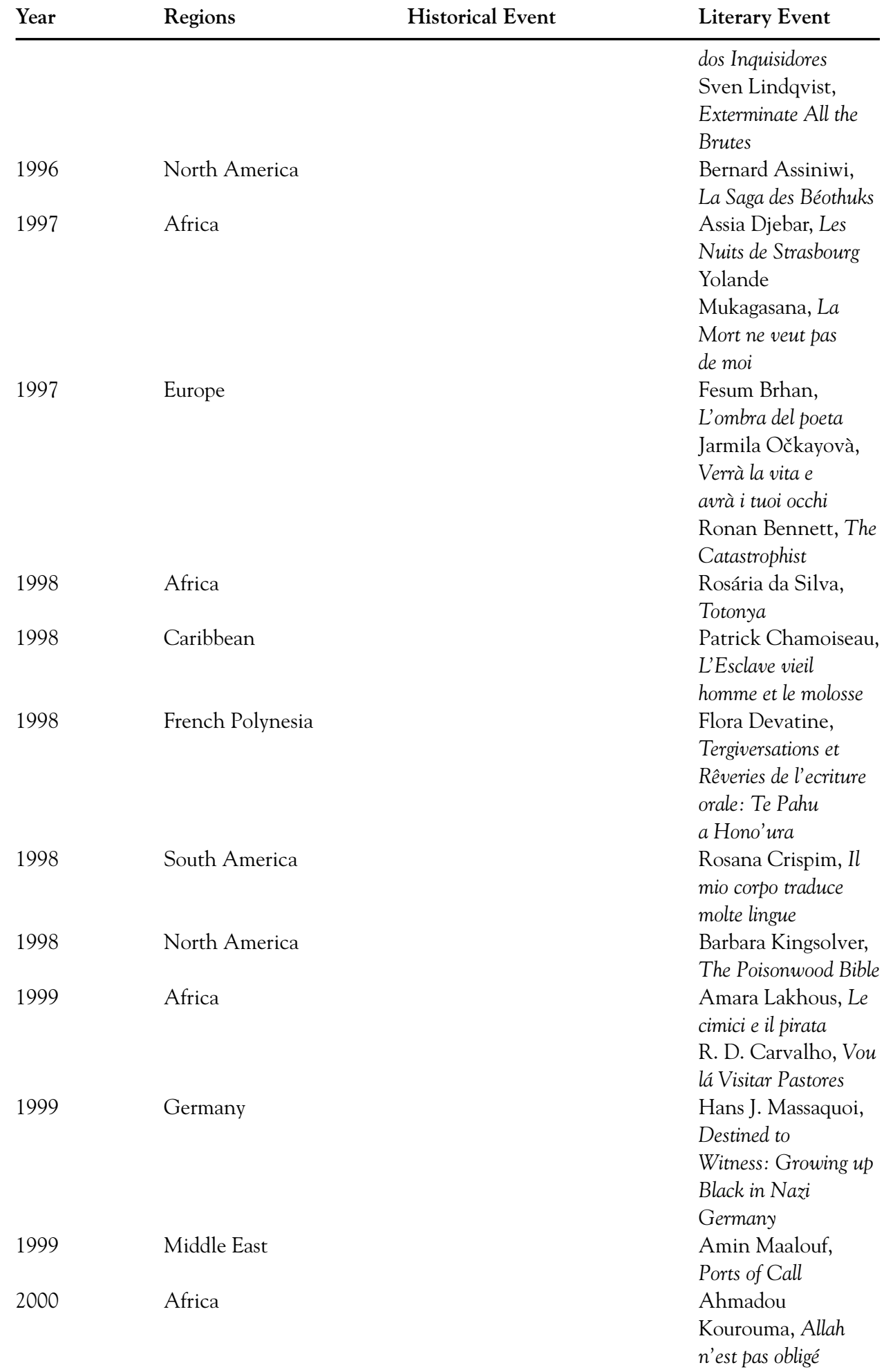




\begin{tabular}{|c|c|c|c|}
\hline Year & Regions & Historical Event & Literary Event \\
\hline & & & $\begin{array}{l}\text { Smari Abdel Malek, } \\
\text { Fiamme in paradiso }\end{array}$ \\
\hline \multirow[t]{2}{*}{2000} & Asia & & Luís Cardoso, The \\
\hline & & & $\begin{array}{l}\text { Crossing: A Story } \\
\text { of East Timor } \\
\text { (Portuguese) }\end{array}$ \\
\hline 2000 & Caribbean & & $\begin{array}{l}\text { Yanick Lahens, Dans } \\
\text { la maison du père }\end{array}$ \\
\hline \multirow[t]{2}{*}{2000} & Europe & & $\begin{array}{l}\text { Gëzin Hadjdari, } \\
\text { Antologia della } \\
\text { pioggia }\end{array}$ \\
\hline & & & $\begin{array}{l}\text { Ron Kubati, Vae } \\
\text { non torna }\end{array}$ \\
\hline 2000 & Middle East & The Camp David Summit & \\
\hline \multirow[t]{6}{*}{2001} & Africa & & Bahassan Adamodjy, \\
\hline & & & Milandos de um \\
\hline & & & Sonho \\
\hline & & & Lília Momplé, \\
\hline & & & Neighbours: The \\
\hline & & & $\begin{array}{l}\text { Story of a Murder } \\
\text { (Portuguese) }\end{array}$ \\
\hline \multirow[t]{2}{*}{2001} & Europe & & Alejandro Leiva \\
\hline & & & Wenger, Till vår ära \\
\hline \multirow[t]{2}{*}{2001} & France/Africa & Loi Taubira, recognising & Fatou Diome, $L a$ \\
\hline & & $\begin{array}{l}\text { slavery as a crime against } \\
\text { humanity }\end{array}$ & Préférence nationale \\
\hline \multirow[t]{2}{*}{2001} & Italy/Africa & & Jadelin Mabiala \\
\hline & & & $\begin{array}{l}\text { Gangbo, Verso la } \\
\text { notte BAKONKA }\end{array}$ \\
\hline \multirow[t]{2}{*}{2001} & Middle East & & Muin Madih Masri, \\
\hline & & & Il sole d'inverno \\
\hline \multirow[t]{8}{*}{2002} & Africa & & José Eduardo \\
\hline & & & Agualusa, Creole \\
\hline & & & (Portuguese) \\
\hline & & & Kossi Komla-Ebri, \\
\hline & & & Imbarrazzismi I \\
\hline & & & Paulina Chiziane, \\
\hline & & & Niketche. Uma \\
\hline & & & História de Poligamia \\
\hline 2002 & Middle East & $\begin{array}{l}\text { Israel launches a major military } \\
\text { offensive in the West Bank }\end{array}$ & \\
\hline \multirow[t]{2}{*}{2003} & Africa & & Mbacke Gadji, \\
\hline & & & $\begin{array}{l}\text { Kelefa: la prova del } \\
\text { pozzo }\end{array}$ \\
\hline \multirow[t]{4}{*}{2003} & Europe & & Fatou Diome, Le \\
\hline & & & Ventre de \\
\hline & & & l'Atlantique \\
\hline & & & $\begin{array}{l}\text { Gerhard Seyfried, } \\
\text { Herero }\end{array}$ \\
\hline
\end{tabular}




\begin{tabular}{|c|c|c|c|}
\hline Year & Regions & Historical Event & Literary Event \\
\hline & & & $\begin{array}{l}\text { Igiaba Scego, La } \\
\text { nomade che amava } \\
\text { Alfred Hitchcock }\end{array}$ \\
\hline 2003 & New Caledonia & & $\begin{array}{l}\text { Nicolas Kurtovitch, } \\
\text { Le Piéton du dharma }\end{array}$ \\
\hline 2003 & Turkey & & $\begin{array}{l}\text { Orhan Pamuk's My } \\
\text { Name is Red wins } \\
\text { the IMPACV } \\
\text { Dublin Literary } \\
\text { Award }\end{array}$ \\
\hline 2004 & Africa & & $\begin{array}{l}\text { J. M. G. Le Clézio, } \\
\text { L'Africain } \\
\text { Werewere Liking, } \\
\text { La Mémoire amputée }\end{array}$ \\
\hline 2004 & Europe & & $\begin{array}{l}\text { Jonas Hassen } \\
\text { Khemiri, Ett öga rött }\end{array}$ \\
\hline 2004 & New Caledonia & & $\begin{array}{l}\text { Pierre Gope, } \mathrm{La} \\
\text { Parenthèse }\end{array}$ \\
\hline 2004 & South America & & $\begin{array}{l}\text { Christiana de } \\
\text { Caldas Brito, Qui e } \\
\text { là racconti }\end{array}$ \\
\hline 2004 & Vietnam/France & & Linda Lê, Kriss \\
\hline 2005 & Middle East & $\begin{array}{l}\text { Israel completes unilateral } \\
\text { disengagement plan in the } \\
\text { Gaza Strip }\end{array}$ & \\
\hline 2005 & Europe & $\begin{array}{l}\text { In France, the controversial } 23 \\
\text { February law includes a clause } \\
\text { obliging educators to teach } \\
\text { the benefits of colonisation }\end{array}$ & $\begin{array}{l}\text { Jeff Janoda, Saga: } \\
\text { A Novel of } \\
\text { Medieval Iceland } \\
\text { Marjaneh Bakhtiari, } \\
\text { Kalla det vad fan } \\
\text { du vill }\end{array}$ \\
\hline 2005 & Vietnam/France & & $\begin{array}{l}\text { Linda Lê, Le } \\
\text { Complexe de Caliban }\end{array}$ \\
\hline 2006 & Africa & & $\begin{array}{l}\text { Aminata Fofana, La } \\
\text { luna che mi seguiva } \\
\text { Mia Couto, A } \\
\text { Sleepwalking Land } \\
\text { (1992, Portuguese) }\end{array}$ \\
\hline 2006 & Europe & & $\begin{array}{l}\text { Jonas Hassen } \\
\text { Khemiri, Montecore: } \\
\text { En unik tiger }\end{array}$ \\
\hline 2006 & Middle East & $\begin{array}{l}\text { The militant Hamas party wins } \\
\text { legislative elections; } \\
\text { a UN study reports the plight } \\
\text { of inhabitants in the Gaza } \\
\text { Strip as 'intolerable' }\end{array}$ & $\begin{array}{l}\text { Orhan Pamuk wins } \\
\text { the Nobel Prize for } \\
\text { Literature }\end{array}$ \\
\hline
\end{tabular}

